\title{
Effect of sample geometry on the phase boundary of a mesoscopic superconducting loop
}

\author{
G. R. Berdiyorov, ${ }^{1,2}$ S. H. Yu, ${ }^{2}$ Z. L. Xiao, ${ }^{2,3}, *$ F. M. Peeters, ${ }^{1, \dagger}$ J. Hua, ${ }^{2,3}$ A. Imre, ${ }^{3,4}$ and W. K. Kwok ${ }^{3}$ \\ ${ }^{1}$ Departement Fysica, Universiteit Antwerpen, Groenenborgerlaan 171, B-2020 Antwerpen, Belgium \\ ${ }^{2}$ Department of Physics, Northern Illinois University, DeKalb, Illinois 60115, USA \\ ${ }^{3}$ Materials Science Division, Argonne National Laboratory, Argonne, Illinois 60439, USA \\ ${ }^{4}$ Center for Nanoscale Materials, Argonne National Laboratory, Argonne, Illinois 60439, USA
}

(Received 18 May 2009; published 17 August 2009)

\begin{abstract}
We studied the effect of sample geometry on the evolution of the superconducting state in nanoscale $\mathrm{Nb}$ circular and square loops by transport measurements. A multistage resistive transition with temperature is found for both samples, which is related to the effect of contact leads made from the same superconducting material. The $H-T$ phase diagrams close to $T_{c 0}$ show clear periodic oscillations on top of a parabolic background, i.e., Little-Parks effect. However, the amplitude of the oscillations decreases faster in the circular loop compared to the one in the square sample. Numerical simulations are conducted within the nonlinear GinzburgLandau theory to show the effect of sample geometry on the nucleation of superconductivity in superconducting loop structures.
\end{abstract}

DOI: 10.1103/PhysRevB.80.064511

PACS number(s): 74.78.-w, 73.23.-b, 74.40.+k, 85.25.-j

\section{INTRODUCTION}

Properties of superconducting vortices in mesoscopic superconductors of size compared to characteristic length scales (magnetic penetration depth $\lambda$ and the superconducting coherence length $\xi$ ) are considerable different from the ones in bulk superconductors. Sample boundary imposes its geometry on the vortex distribution and, therefore, different geometries favor different arrangement of vortices. In small systems, vortices may overlap so strongly that it is more favorable to form multiquanta giant vortices. The existence of giant vortex states and transition from the giant vortex state to multivortex states have already been predicted theoretically ${ }^{1}$ and confirmed experimentally using multiplesmall-tunnel-junction method. ${ }^{2}$ It was also found that the influence of boundaries can lead to stabilization of the vortexantivortex molecules, which preserve the symmetry of the sample. ${ }^{3}$ However, such molecules have a very shallow minimum in the free energy $y^{4,5}$ and are very sensitive to the change of the sample shape. ${ }^{6}$ Recent theoretical calculations within the Ginzburg-Landau theory showed that these symmetry-induced vortex-antivortex molecules can be enforced by artificial pining centers ${ }^{7}$ or by a magnetic dot on top of the sample, ${ }^{8}$ with their diagnostic features enhanced by orders of magnitude.

The critical parameters of mesoscopic superconductors are also influenced by the vortex structure inside the sample. ${ }^{9,10}$ As was first shown by Saint-James, ${ }^{11}$ each new vortex entry brings an oscillation in the $T_{c}(H)$ phase boundary of a superconducting disk, similar to Little-Parks effect in thin walled loops. ${ }^{12}$ However, in the former case, the period of $T_{c}(H)$ is not constant and decreases with increasing the applied field. The properties of superconducting samples in these two extreme cases have been further investigated both theoretically ${ }^{1}$ and experimentally. ${ }^{13}$

The intermediate case-a superconducting circular loop with finite width - has also been investigated in the past. For example, Bardeen ${ }^{14}$ showed within the Ginzburg-Landau (GL) theory that the flux is quantized in units of $\nu \Phi_{0}$ (with $\nu<1$ and $\Phi_{0}=h c / 2 e$ is flux quantum) in cylinders of very small diameters and with a wall thickness of the order of the penetration depth. Groff and Parks showed theoretically that in the circular loop, the flux through an area $S=\pi r_{m}^{2}$ is quantized in units of $\Phi_{0}$, with $r_{m}=\left(r_{i}+r_{o}\right) / 2$ the arithmetic mean of the inner $\left(r_{i}\right)$ and outer $\left(r_{o}\right)$ radii. ${ }^{15}$ This effective radius was also predicted to be calculated as $r_{e f f}=\sqrt{r_{i} r_{o}} .{ }^{16}$ These two different values $r_{m}$ and $r_{\text {eff }}$ are nearly identical for a narrow ring. Numerical simulations within the GL theory ${ }^{17}$ showed that the value of $r_{i}<r_{e f f}<r_{o}$ depends on the vorticity $L$ and in fact $r_{\text {eff }}$ turns out to be an oscillating function of the magnetic field. It was also found that ${ }^{18}$ with decreasing the width of the superconducting loop, the disklike behavior of $T_{c}(H)$ with a quasilinear background [characteristic of threedimensional (3D) behavior] is shown to evolve into parabolic $T_{c}(H)$ background, superimposed with perfectly periodic oscillations. Morelle et al., ${ }^{19}$ studied using transport measurements the nucleation of superconductivity in a circular loop for different values of the inner radius $r_{i}$. They also found the transition from a one-dimensional to a twodimensional regime at larger magnetic field for smaller $r_{i}$. A parabolic background superimposed with periodic oscillations was found in the $T_{c}(H)$ phase boundary for thinner loops. At larger magnetic field, the loops recover the behavior of the disk without a hole in the center, which was explained by the formation of giant vortex states. In an external flux near $\Phi_{0} / 2$, a superconducting loop behaves like an Ising spin due to the degenerate energy states with opposite direction of the supercurrents. Davidovic et al. ${ }^{20}$ showed that an array of such loops can act as a model for a two-dimensional Ising antiferromagnet.

Oscillations in the $T_{c}(H)$ phase boundary have also been observed experimentally in superconducting square loops with very thin width. ${ }^{13,21}$ From the previous theoretical works, we know that the nucleation of the superconducting state is substantially enhanced in samples with sharp corners due to the locally enhanced superconducting order parameter near the corners. ${ }^{5,22,23}$ However, in the limiting case of very thin loops, the distribution of the superconducting order pa- 


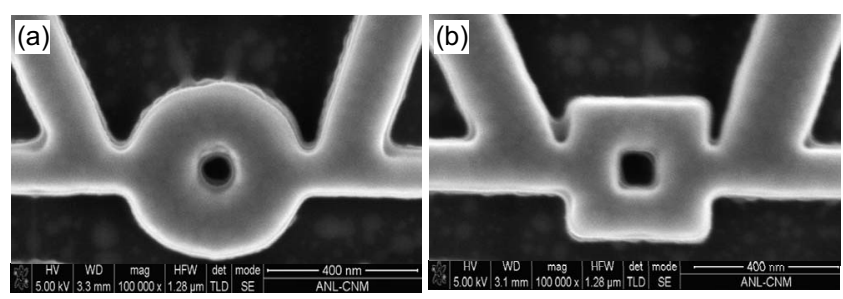

FIG. 1. SEM images of (a) a circular loop (with outer radius $r_{o}=235 \mathrm{~nm}$, inner radius $r_{i}=55 \mathrm{~nm}$, and current contact width $165 \mathrm{~nm}$ ) and (b) a square loop (with size $l=370 \mathrm{~nm}$, hole size $a=130 \mathrm{~nm}$, and current contact width $200 \mathrm{~nm}$ ). The thickness of both samples is $t=100 \mathrm{~nm}$.

rameter is uniform along the sample and the effect of the sample geometry becomes less important. To our knowledge, there are no theoretical and experimental works devoted to study the effect of the sample geometry on the fluxoid quantization in superconducting loops with intermediate width. Therefore, in this paper, we conduct comparative study of $T_{c}(H)$ phase boundary in superconducting square and circular loops by means of transport measurements. We show that the $T_{c}(H)$ phase diagram of both samples exhibits periodic oscillations, similar to the Little-Parks effect. However, the amplitude of oscillations is smaller in the circular sample compared to the one in the square sample, which is due to the geometry of the sample. Our numerical simulations within the GL theory confirm the experimental findings.

The paper is organized as follows. In Sec. II, we present sample fabrication and measurement techniques. Experimental results together with numerical simulations within the GL theory are presented in Secs. III and IV to investigate the properties of resistance transitions and the superconducting/ normal phase boundary. Conclusions are given in Sec. V.

\section{SAMPLE CHARACTERISTICS AND MEASUREMENT TECHNIQUE}

Superconducting niobium square and circular loops (Fig. 1) were patterned out of $t=100 \mathrm{~nm}$ thick niobium microbridges with focused-ion-beam (FIB) milling. First, a 300nm-thick $E$-beam resist layer, polymethil methacrylate (PMMA-950-A4), was spin coated on silicon substrate with a 100-nm-thick $\mathrm{SiO}_{2}$ insulator layer. Then, the sample was baked on a hot plate at $180{ }^{\circ} \mathrm{C}$ for $90 \mathrm{~s}$. A pattern of a 4 by $10 \mu \mathrm{m}$ bridge with standard four-probe transport measurement contacts leads was used for exposure by Raith 150 $E$-beam machine. Following a development in methyl isobutyl ketone (MIBK) and isopropyl alcohol (IPA) (1:3) solution for $75 \mathrm{~s}$ and washed with isopropanol, the designed pattern was transformed onto the wafer. A 100-nm-thick niobium was then deposited on the wafer with an AJA ATC2400 sputtering system. The film thickness was determined by a calibrated quartz-crystal thickness monitor during the deposition. The pressure of the working argon gas was maintained at $1.5 \mathrm{mTorr}$ during sputtering. The growth rate was $3.1 \AA /$ s. After deposition, a standard lift-off procedure using acetone was followed, leading to only the niobium bridge structure left on the wafer. A square loop and circular loop

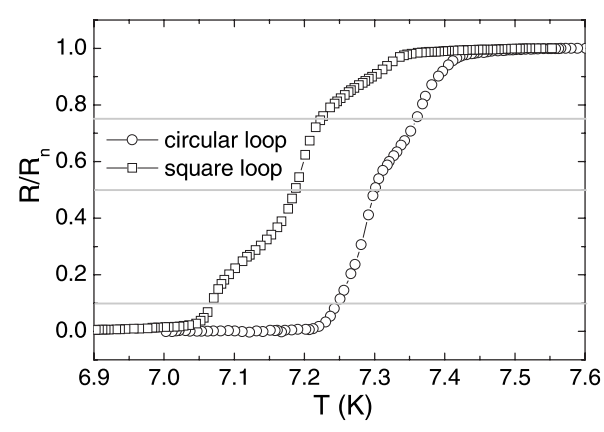

FIG. 2. The resistance (normalized to the normal-state resistance) $R / R_{n}$ as a function of temperature $T$ for the circular (circular symbols) and square (square symbols) samples at zero magnetic field. The resistance criteria, which was used to construct the $T_{c}(H)$ phase diagrams for the loops, are indicated by horizontal gray lines (see text for discussions).

with a hole in each of them were milled with a FEI Nova 600 FIB machine in the middle part of the bridge. A gallium source was used for producing the ion beam. The accelerated voltage was $30 \mathrm{kV}$ and the beam current was fixed at $28 \mathrm{pA}$. Under this condition, the approximate spot size of the ion beam was $20 \mathrm{~nm}$. The grooves carved by FIB are more than $150 \mathrm{~nm}$ wide to avoid any effects coming from surrounding superconducting material. We used the combination of $E$-beam patterning and FIB milling instead of direct $E$-beam patterning of the structures to avoid $E$-beam resist contaminations which degrade the quality of the $\mathrm{Nb}$ nanostructures. More details on the sample fabrication can be found in Ref. 24.

Transport measurements were carried out in a physics property measurement system (PPMS) (from Quantum Design) by using a standard four-probe dc setup. The magnetic field is always applied perpendicular to the system. By horizontally rotating the sample, the angle between the surface of the sample and the magnetic field can be precisely controlled. The range of the rotation is from $-10^{\circ}$ to $370^{\circ}$ with resolution $\sim 0.053^{\circ}$. The $T_{c}(H)$ phase boundary is determined from a set of resistance vs temperature $R(T)$ curves measured at various magnetic fields using a certain resistance criterion $R_{c}$.

\section{RESISTANCE TRANSITIONS}

Figure 2 shows the resistance versus temperature $R(T)$ curves for circular (circular symbols) and square (square symbols) loops at zero magnetic field. The driving current is $10 \mu \mathrm{A}$. It is seen from this figure that the resistance of both samples shows several transition stages. For the circular loop, the resistance drops sharper at high temperatures, followed by slow decrease of the resistance as temperature decreases. This can be understood from the width dependence of the critical temperature of the sample. ${ }^{24}$ As we see from the SEM image of the sample [Fig. 1(a)], the width of the circular loop is larger than the width of the contacts. Therefore, superconductivity first nucleates at the loop itself (first drop of the resistance) and later the contacts become superconducting. In the case of the square loop (square symbols in 



FIG. 3. (Color online) Theoretically calculated time-averaged voltage $V$ as a function of temperature $T$ for the sample illustrated in the inset of (a). The size of the superconducting loop is $l=370 \mathrm{~nm}$, thickness is $t=100 \mathrm{~nm}$, the hole size is $a=130 \mathrm{~nm}$, the contact length is $l_{n}=370 \mathrm{~nm}$, and the widths of the contact are $d=100 \mathrm{~nm}$ (solid curves) and $d=300 \mathrm{~nm}$ (dashed curves). The results are obtained for the driving current $20 \mu \mathrm{A}$ and the applied magnetic fields $H=0$ (thick curves) and $H=200 \mathrm{G}$ (thin curves). The contour plots (1-8) show the Cooper-pair density at temperatures indicated in (a) for $H=0(1,2,5,6)$ and $H=200 \mathrm{G}(3,4,7,8)$.

Fig. 2), the $R(T)$ curve is characterized by a slowly decreasing resistance at high temperatures arising from the nucleation of superconductivity in the contacts, followed by a sharper drop of the resistance, which corresponds to the transition of the loop into superconducting state. That is, since the loop width to contact size ratio is different in two samples which results in multistage transitions, therefore, different criteria should be used to define the critical temperature of the loops.

The difference in the behavior of $R(T)$ curves in the above samples can also be explained by the difference in the transport current density across the sample. In what follows, we perform numerical simulations within the time-dependent Ginzburg-Landau (TDGL) theory to study the transport properties of superconducting loops. We consider a square superconductor (size $l=370 \mathrm{~nm}$, thickness $t=100 \mathrm{~nm}$ ) containing a square hole in the middle (size $a=130 \mathrm{~nm}$ ). The magnet field is applied perpendicular to the sample and the electric current is applied through the current contacts (length $l_{n}=370 \mathrm{~nm}$ and width $d$ ) [see the inset of Fig. 3(a)]. For this system, we solved the TDGL equation ${ }^{25}$

$$
\begin{array}{r}
\frac{u}{\sqrt{1+\Gamma^{2}|\psi|^{2}}}\left(\frac{\partial}{\partial t}+i \varphi+\frac{\Gamma^{2}}{2} \frac{\partial|\psi|^{2}}{\partial t}\right) \psi \\
=(\nabla-i \mathbf{A})^{2} \psi+\left(1-T-|\psi|^{2}\right) \psi
\end{array}
$$

coupled with the equation for the electrostatic potential $\Delta \varphi=\operatorname{div}\left(\Im\left[\psi^{*}(\nabla-i \mathbf{A}) \psi\right]\right)$. We assume a superconductor with a good thermal contact with the substrate so that we do not have to couple the TDGL equations with the heat equation. Here, distances are scaled to coherence length $\xi$, time is in units of $\tau_{\mathrm{GL}}=\pi \hbar / 8 k_{B} T u$, the electrostatic potential $\varphi$ is given in $\varphi_{0}=\hbar / 2 e \tau_{\mathrm{GL}}$, and vector potential $\mathbf{A}$ is scaled by $H_{c 2} \xi$. The parameter $\Gamma=2 \tau_{E} \Psi_{0} / \hbar$ characterizes the chosen superconducting material (with $\tau_{E}$ being the inelastic electroncollision time). In the present simulations, we take $\xi(0)=10 \mathrm{~nm}$ and $\lambda(0)=200 \mathrm{~nm}$ (typical values for thin $\mathrm{Nb}$ films $\left.{ }^{26}\right)$. The parameter $u$ is equal to 5.79 in accordance with Ref. 25 and we used $\Gamma=10$, which is typical for $\mathrm{Nb}$ samples in the range of applicability of the TDGL theory due to small $\tau_{E}$. We limit ourselves to the case when the effect of the self-induced magnetic field is negligible. Neumann boundary conditions are taken at all sample boundaries, except at the end of the leads where we use $\psi=0$ and $\left.\nabla \varphi\right|_{n}=-j$ conditions.

It was shown in Ref. 27 that the presence of voltage and current leads has an influence on the results of transport measurements in mesoscopic superconductors and depending on the size and shape of the contacts may lead to a resistance anomaly close to $T_{c}$. Numerical simulations within the TDGL theory also show the effect of the current contacts on the critical parameters of the sample. ${ }^{28}$ To see the effect of current contacts on the properties of our square loop sample, we plotted in Fig. 3(a) time-averaged voltage of the sample as a function of temperature for two different values of the contact width $d$. For $d=100 \mathrm{~nm}$ (solid curves), which is smaller than the width of the loop $w=(l-a) / 2=120 \mathrm{~nm}$, when starting from the normal state and decreasing the temperature, the loop first becomes superconducting (1) followed by the nucleation of superconductivity at the contacts (2). The latter is due to the larger current density at the contact leads. Both of these transitions bring a finite jump in the output voltage [see thick solid curve in Fig. 3(a)], i.e., a similar multistage transition is seen in the experimental $V(T)$ curve (see Fig. 2). The same behavior in $V(T)$ is found for nonzero magnetic field $(3,4)$ with the only difference that the transition points shift to lower temperatures (thin solid curve). Note that the finite voltage at lower temperatures is due to superconducting/normal boundaries at the ends of the contact leads. In the case of larger contact width (larger than the width of the loop) superconductivity first nucleates at the contacts (5) with decreasing temperature from $T_{c 0}$. The loop itself is in the normal state via the appearance of phase slip lines (5). ${ }^{28}$ With further decreasing the temperature, the whole sample becomes superconducting (6). At nonzero magnetic fields, the phase slip lines convert themselves into a flow of (kinematic) vortices (7) (Ref. 29) and vortices can 

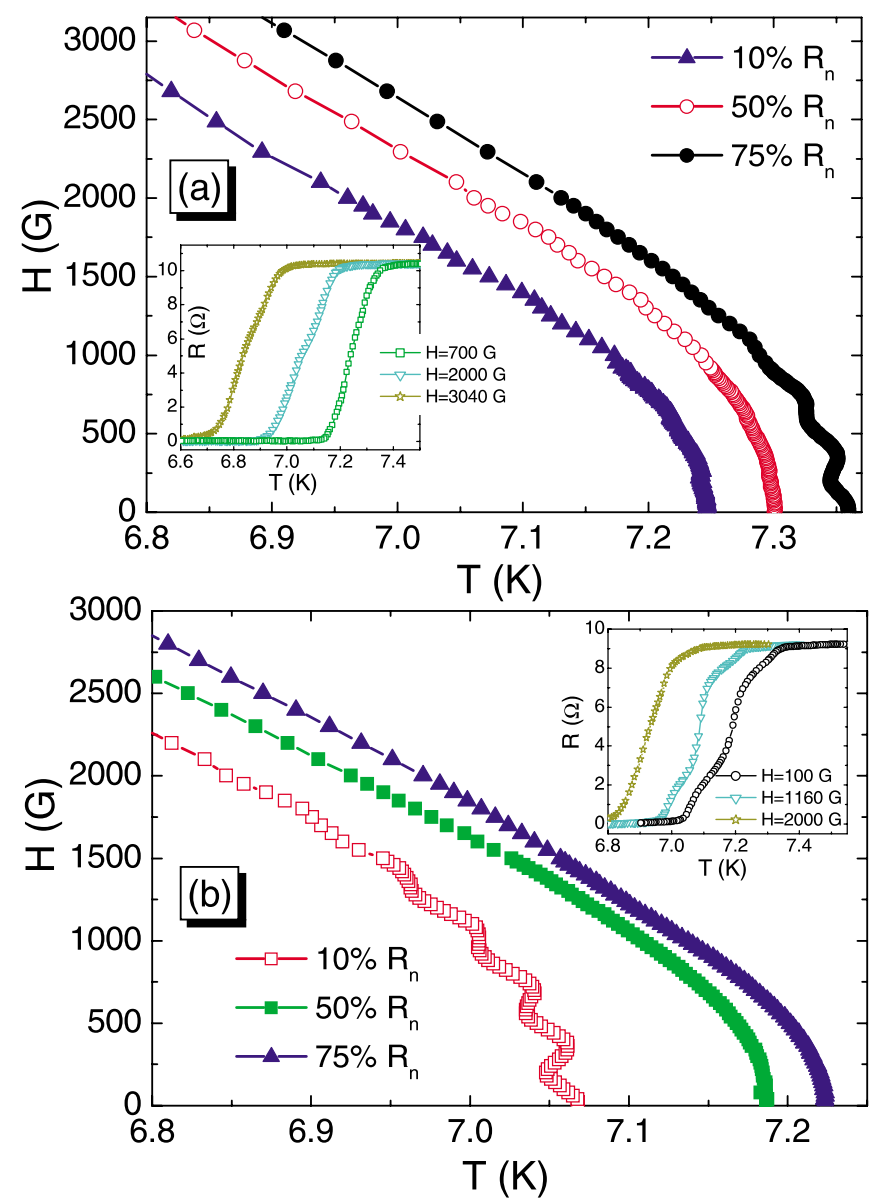

FIG. 4. (Color online) $T_{c}(H)$ phase diagrams of the (a) circular and (b) square loops obtained with different transition criteria: $75 \%$ $R_{n}, 50 \% R_{n}$, and $10 \% R_{n}$. Insets show the resistive transitions $R(T)$ in different applied fields. The driving current in both cases is $10 \mu \mathrm{A}$.

be trapped inside the hole at small temperatures (8). The $V(T)$ curve [dashed curves in Fig. 3(a)] also shows multistage transitions as in the case of the smaller contact width (solid curves) with the difference that the temperature range between the transitions becomes smaller. Thus, multistage transitions in the experimentally obtained $R(T)$ curves are due to the nucleation of superconductivity in different parts of the sample. Therefore, the constructed superconducting/ normal phase boundary strongly depends on chosen resistance criterion.

\section{IV. $T_{c}(H)$ PHASE DIAGRAM}

As shown in the insets of the figure, multistage transition is still preserved in the $R(T)$ curves in the presence of the magnetic field, which is due to the contacts made from the same material as the sample itself. Therefore, the $T_{c}(H)$ phase diagram will also depend on the transition criterion. Figure 4 shows the experimental $T_{c}(H)$ phase boundary of the samples constructed using three different criteria of superconducting-normal transition resistance $R_{n}$. As demonstrated in Fig. 4(a), for the case of the circular loop, although

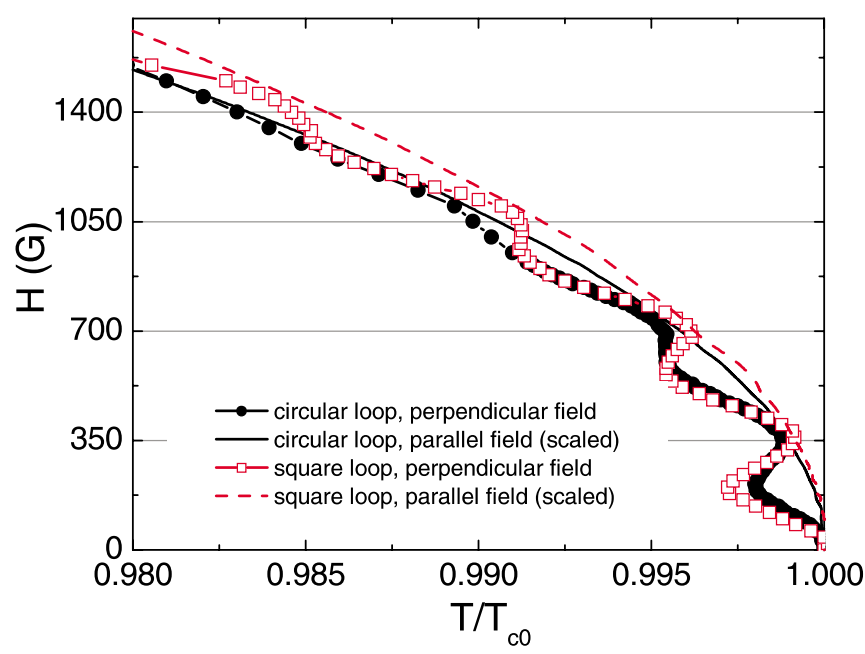

FIG. 5. (Color online) $H-T$ phase diagrams of the circular (circles) and the square (squares) loops. Solid (dashed) curve is the result obtained in parallel magnetic field for the circular (square) loop, scaled with a scaling factor $0.54(0.56)$. The phase boundary is determined at $75 \%$ and $10 \%$ of the normal-state resistance, $R_{n}$, for circular and square samples, respectively.

all three criteria give the same parabolic background (typical for thin stripes in perpendicular field), periodic oscillations are obtained only when we use $75 \% R_{n}$ criterion (solid circles). However, in the case of the square sample [Fig. 4(b)], the parabolic background, superimposed on top of a periodic oscillation, is obtained by $10 \% R_{n}$ criterion (open squares). As mentioned in the preceding section, in order to observe the superconducting/normal boundary of the loop (instead of the leads), we need to use a different resistance criterion for loops with different contact sizes. Therefore, the usual $50 \% R_{n}$ criterion cannot be applied to our measured systems. Fitting the linear part of the phase diagrams, we obtain the coherence lengths of $\xi(0)=9.42 \mathrm{~nm}$ and $\xi(0)=10.86 \mathrm{~nm}$ for the circular and square samples, respectively, which are typical for $\mathrm{Nb}$ thin films. ${ }^{26}$

Figure 5 shows the $H-T$ phase diagrams (in normalized temperature) of the circular and square samples determined at $75 \%$ and $10 \%$ of the normal-state resistance, $R_{n}$, respectively. As we mentioned above, the phase diagrams of both samples show clear oscillations with period $\sim 350 \mathrm{G}$ superimposed on top of a parabolic background. However, the amplitude of the oscillations vanishes faster in the circular loop (solid circles) compared to square loop (open squares): only first two peaks are clearly seen in the case of circular loop, while for the square sample, fourth peak is still visible. Second, the transition from parabolic to (quasi-)linear regime takes place at higher temperatures for the circular sample.

Since the width $w$ and the thickness $t$ of the loops become comparable to $\xi(T)$ close to $T_{c 0}$, the parabolic background of the $H$-T curve in the perpendicular field should coincide with the one obtained in the parallel field by multiplying the latter with a scaling factor $t / w$ (Refs. 24 and 30). Solid and dashed curves in Fig. 5 show the scaled $H$ - $T$ curves of the circular and square loops, respectively, in parallel magnetic field. The scaling factors are 0.54 (for the circular loop) and 0.56 (for the square sample). The scaling factor for the circular loop is 

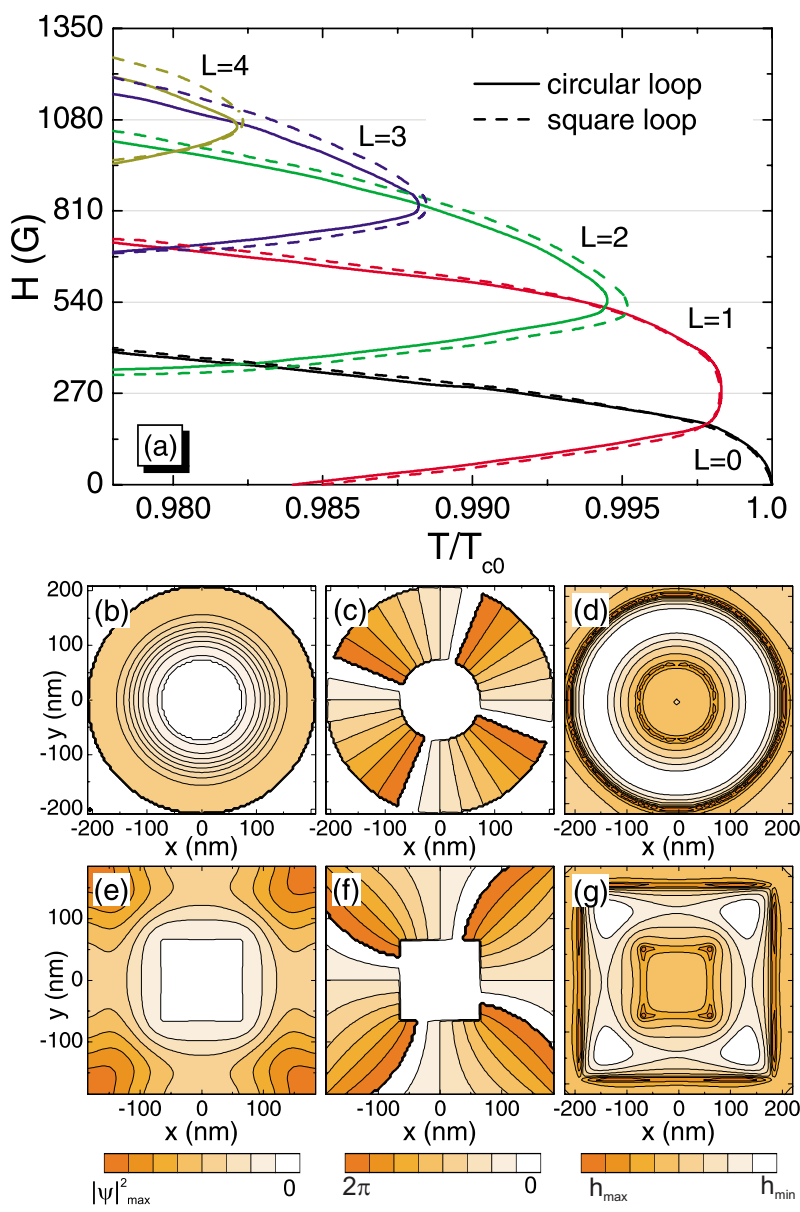

FIG. 6. (Color online) (a) $H-T$ phase diagrams of the circular (solid curves) and square (dashed curves) loops. The size of the square sample is $l=370 \mathrm{~nm}$, the size of the hole is $a=130 \mathrm{~nm}$, and the thickness is $t=100 \mathrm{~nm}$. The circular sample has the same superconducting area as the square sample, i.e., $r_{o}=l / \sqrt{\pi}, r_{i}=a / \sqrt{\pi}$, and $t=100 \mathrm{~nm}$. Contour plots of the [(b) and (e)] Cooper-pair density, $[(\mathrm{c})$ and (f) $]$ phase of the order parameter, and $[(\mathrm{d})$ and $(\mathrm{g})]$ the magnetic field distribution for the $[(\mathrm{b})-(\mathrm{d})]$ circular and $[(\mathrm{e})-(\mathrm{g})]$ square loops at temperature $T=0.98 T_{c 0}$ and in magnetic fields [(b)(d) $] H=1144 \mathrm{G}$ and $[(\mathrm{e})-(\mathrm{g})] H=1205 \mathrm{G}$.

very close to $t /\left(R_{o}-R_{i}\right)=0.556$, whereas in the case of the square sample, the scaling factor is smaller than $2 t /(l-a)=0.83$. The discrepancy in the latter case is due to the larger contact leads.

As we have mentioned in Sec. I, in circular loops with finite width, the magnetic flux through the hole is not quantized in multiples of $\Phi_{0}$, rather it is quantized over the area with effective radius $r_{e f f}$. From the period of oscillations $\Delta H \approx 350 \mathrm{G}$, we find an effective radius $r_{\text {eff }} \approx 140 \mathrm{~nm}$ for the circular sample, which is very close to the arithmetic mean of the outer $r_{o}$ and inner $r_{i}$ radii. For the square loop, the side length of the effective area, $l_{\text {eff }}$, over which the flux is quantized in units of $\Phi_{0}$ equals to $l_{e f f} \approx 247 \mathrm{~nm}$. This value is also very close to the arithmetic mean of the size of the sample and the hole $(l+a) / 2=250 \mathrm{~nm}$. Another formula for $l_{e f f}$ is given in Ref. 20, assuming uniform nucleation, as $l_{\text {eff }}=\sqrt{\left(l^{2}-a^{2}\right) /(2 \ln (l / a))}=239.51 \mathrm{~nm}$. This effective area gives us the field $H_{1}=360.85 \mathrm{G}$, which is slightly larger than the experimentally obtained period of the oscillations in the $H-T$ phase diagram.

To find out whether the difference in the $H-T$ phase diagrams of the two considered samples is due to the sample geometry or because of the contacts, we conducted numerical simulations within the GL theory. For the given samples, we solved two coupled GL equations, which can be written in dimensionless units in the following form:

$$
\begin{gathered}
\frac{\partial \psi}{\partial t}=(-i \vec{\nabla}-\vec{A})^{2} \psi-\psi\left(1-|\psi|^{2}\right), \\
-\Delta \vec{A}=\frac{t}{\kappa^{2}}\left[\frac{1}{2 i}\left(\psi^{*} \vec{\nabla} \psi-\psi \vec{\nabla} \psi^{*}\right)-|\psi|^{2} \vec{A}\right],
\end{gathered}
$$

where $t$ is the sample thickness and $\kappa=\lambda(T) / \xi(T)$ is the GL parameter. The same dimensionless units are used as in Eq. (1). We followed the approach of Ref. 1 to solve Eqs. (2) and (3) self-consistently. Superconducting-vacuum boundary condition $\left.(-i \vec{\nabla}-\vec{A}) \psi\right|_{n}=0$ is used at the inner and outer boundaries and the temperature is included in the calculations through the temperature dependence of the coherence length $\xi(T)=\xi(0) / \sqrt{1-T / T_{c 0}}$ and the penetration depth $\lambda(T)=\lambda(0) / \sqrt{1-T / T_{c 0}}$.

Figure 6(a) shows the calculated $H-T$ phase diagrams for the circular (solid curves) and square (dashed curves) loops. Note that theoretically, we do not calculate the resistance but rather determine for given temperature the magnetic field at which the superconducting state is completely disappeared. Possible difference between theory and experiment is also due to the difference in the criterion to determine the $T_{c}-H$ curves. Moreover, the presence of current (voltage) contacts, which are not taken into account in the present calculations, also may lead to the discrepancy between the theory and the experiment. $^{27}$

The square sample has the same dimensions as the square loop in the experiment [see Fig. 1(b)] and the circular sample has the same superconducting area as the square loop (i.e., $\left.r_{o}=l / \sqrt{\pi}, r_{i}=a / \sqrt{\pi}\right)$. As we see from this figure, the $H-T$ curves of both samples overlap when they are in the Meissner state ( $L=0$ state), i.e., the sample shape does not affect the phase boundary in the Meissner state, as was also shown in preceding works. ${ }^{5,9}$ For the $L=1$ state, a difference is found only far away from the superconducting/normal transition: the penetration of the first vortex in the circular sample occurs at lower magnetic field values and the expulsion takes place at larger magnetic fields for a fixed temperature. For the $L=2$ state, a significant increase of the vortex stability is found for the square sample compared to the circular loop. The difference in the $H-T$ boundary of the two samples becomes more pronounced for the vortex states with larger vorticity $L$. However, this enhancement of the critical parameters is not due to the stability of different vortex states favored by the geometry of the sample as was reported in preceding works ${ }^{5,9}$ because for the considered temperature range, all vortices are located inside the hole. Figures 6(b)-6(g) show the spatial distribution of the Cooper-pair density, the phase of the superconducting order parameter, and local magnetic field distribution in both samples for the 
$L=4$ state near the superconducting/normal transition point. Since all the flux goes through the hole, the superconducting order parameter is uniformly suppressed in the circular loop [Fig. 6(b)] like in the case of a giant vortex in a superconducting disk. ${ }^{2}$ However, in the square loop, larger Cooperpair density is preserved at the corners of the sample [Fig. $6(\mathrm{e})]$ as was shown in previous studies. ${ }^{5,22,23}$ Therefore, we conclude that the increase of the $H-T$ phase boundary in the square loop is due to the enhancement of superconductivity at the sharp corners of the sample. The smaller amplitude of the experimental $T_{c}(H)$ oscillations in circular loop as compared to the square one can also be explained by the latter effect.

\section{CONCLUSIONS}

In conclusion, using transport measurements, we studied the effect of sample geometry and current contacts on the nucleation of superconductivity in mesoscopic circular and square loops. The $R(T)$ curves show multistage transitions which correspond to the nucleation of superconductivity at the contacts and/or at the loop itself. For both samples, the
$T_{c}(H)$ phase boundary shows a parabolic background with periodic oscillations superimposed on top of it. In a circular loop, the amplitude of the oscillations is weaker and the transition to the linear $T(H)$ regime occurs at higher temperature. Numerical simulations within the GL theory show that the difference in the $H$ - $T$ phase boundary in these two samples is due to the enhanced superconductivity at the corners of the superconducting square loop.

\section{ACKNOWLEDGMENTS}

This material is based on research supported by the National Science Foundation (NSF) under Grant No. DMR0605748 and the U.S. Department of Energy Contract No. DE-AC02-06CH11357. The sample fabrication work was supported by the U.S. Department of Energy Award No. DEFG02-06ER46334. The electron-beam and FIB patternings were performed at Argonne's Center for Nanoscale Materials (CNM). The theoretical part of the work is supported by the Flemish Science Foundation (FWO-Vl) and the Belgian Science Policy (IAP). G.R.B. acknowledges support from FWO-Vlaanderen.

\footnotetext{
*zxiao@niu.edu

†rancois.peeters@ua.ac.be

${ }^{1}$ V. A. Schweigert, F. M. Peeters, and P. S. Deo, Phys. Rev. Lett. 81, 2783 (1998).

${ }^{2}$ A. Kanda, B. J. Baelus, F. M. Peeters, K. Kadowaki, and Y. Ootuka, Phys. Rev. Lett. 93, 257002 (2004).

${ }^{3}$ L. F. Chibotaru, A. Ceulemans, V. Bruyndoncx, and V. V. Moshchalkov, Nature (London) 408, 833 (2000); Phys. Rev. Lett. 86, 1323 (2001).

${ }^{4}$ J. Bonca and V. V. Kabanov, Phys. Rev. B 65, 012509 (2001).

${ }^{5}$ B. J. Baelus and F. M. Peeters, Phys. Rev. B 65, 104515 (2002).

${ }^{6}$ A. S. Mel'nikov, I. M. Nefedov, D. A. Ryzhov, I. A. Shereshevskii, V. M. Vinokur, and P. P. Vysheslavtsev, Phys. Rev. B 65, 140503(R) (2002).

${ }^{7}$ R. Geurts, M. V. Milošević, and F. M. Peeters, Phys. Rev. Lett. 97, 137002 (2006).

${ }^{8}$ C. Carballeira, V. V. Moshchalkov, L. F. Chibotaru, and A. Ceulemans, Phys. Rev. Lett. 95, 237003 (2005).

${ }^{9}$ M. Morelle, J. Bekaert, and V. V. Moshchalkov, Phys. Rev. B 70, 094503 (2004).

${ }^{10}$ B. J. Baelus, A. Kanda, N. Shimizu, K. Tadano, Y. Ootuka, K. Kadowaki, and F. M. Peeters, Phys. Rev. B 73, 024514 (2006).

${ }^{11}$ D. Saint-James, Phys. Lett. 15, 13 (1965).

${ }^{12}$ W. A. Little and R. D. Parks, Phys. Rev. Lett. 9, 9 (1962); R. D. Parks and W. A. Little, Phys. Rev. 133, A97 (1964).

${ }^{13}$ V. V. Moshchalkov, L. Gielen, C. Strunk, R. Jonckheere, X. Qiu, C. Van Haesendonck, and Y. Bruynseraede, Nature (London) 373, 319 (1995).

${ }^{14}$ J. Bardeen, Phys. Rev. Lett. 7, 162 (1961).

${ }^{15}$ R. P. Groff and R. D. Parks, Phys. Rev. 176, 567 (1968).

${ }^{16}$ R. M. Arutunian and G. F. Zharkov, J. Low Temp. Phys. 52, 409 (1983).
}

${ }^{17}$ B. J. Baelus, F. M. Peeters, and V. A. Schweigert, Phys. Rev. B 61, 9734 (2000).

${ }^{18}$ V. Bruyndoncx, L. Van Look, M. Verschuere, and V. V. Moshchalkov, Phys. Rev. B 60, 10468 (1999).

${ }^{19}$ M. Morelle, D. S. Golubovic, and V. V. Moshchalkov, Phys. Rev. B 70, 144528 (2004).

${ }^{20}$ D. Davidović, S. Kumar, D. H. Reich, J. Siegel, S. B. Field, R. C. Tiberio, R. Hey, and K. Ploog, Phys. Rev. B 55, 6518 (1997).

${ }^{21}$ C. Strunk, V. Bruyndoncx, V. V. Moshchalkov, C. Van Haesendonck, Y. Bruynseraede, and R. Jonckheere, Phys. Rev. B 54, R12701 (1996).

${ }^{22}$ V. M. Fomin, J. T. Devreese, and V. V. Moshchalkov, Europhys. Lett. 42, 553 (1998); 46, 118 (1999).

${ }^{23}$ V. A. Schweigert and F. M. Peeters, Phys. Rev. B 60, 3084 (1999).

${ }^{24}$ J. Hua, Z. L. Xiao, A. Imre, S. H. Yu, U. Patel, L. E. Ocola, R. Divan, A. Koshelev, J. Pearson, U. Welp, and W. K. Kwok, Phys. Rev. Lett. 101, 077003 (2008).

${ }^{25}$ L. Kramer and R. J. Watts-Tobin, Phys. Rev. Lett. 40, 1041 (1978).

${ }^{26}$ A. I. Gubin, K. S. Ílin, S. A. Vitusevich, M. Siegel, and N. Klein, Phys. Rev. B 72, 064503 (2005).

${ }^{27}$ M. Morelle, Y. Bruynseraede, and V. V. Moshchalkov, Phys. Status Solidi B 237, 365 (2003).

${ }^{28}$ D. Y. Vodolazov, F. M. Peeters, M. Morelle, and V. V. Moshchalkov, Phys. Rev. B 71, 184502 (2005).

${ }^{29}$ G. R. Berdiyorov, M. V. Milosevic, and F. M. Peeters, Phys. Rev. B 79, 184506 (2009).

${ }^{30}$ U. Patel, Z. L. Xiao, J. Hua, T. Xu, D. Rosenmann, V. Novosad, J. Pearson, U. Welp, W. K. Kwok, and G. W. Crabtree, Phys. Rev. B 76, 020508(R) (2007). 